\title{
Risco para lesão por fricção em idosos longevos na atenção primária à saúde
}

\author{
Risk for friction injury in long-lived elderly in primary health care
}

Riesgo de lesión por fricción en adultos mayores longevos en la atención primaria de salud

Francisco Reis Tristão ${ }^{1}$, Juliana Balbinot Reis Girondi², Karina Silveira de Almeida Hammerschmidt², Cilene Fernandes Soares 3 , Tatiana Martins ${ }^{4}$, Daniella Karine de Souza Lima ${ }^{4}$

ORCID IDS

Tristão FR (D) https://orcid.org/0000-0003-1451-4566

Girondi JBR (D) https://orcid.org/0000-0003-0271-259X

Hammerschmidt KSA (D) https://orcid.org/0000-0002-7140-3427

Soares CF (iD https://orcid.org/0000-0002-6032-8644

Martins T (ID https://orcid.org/0000-0002-2475-0653

Lima DKS (iD https://orcid.org/0000-0001-7167-0907

\section{COMO CITAR}

Tristão FR, Girondi JBR, Hammerschmidt KSA, Soares CF, Martins T, Lima DKS. Risco para lesão por fricção em idosos longevos na atenção primária à saúde. ESTIMA, Braz. J. Enterostomal Ther., 16: e3218. https://doi.org/10.30886/estima.v16.614 PT.

Manuscrito extraído do resultado de monografia intitulada "Estratificação de risco para ocorrência de lesão por fricção e lesão por pressão em idosos na comunidade", de autoria de Francisco Reis Tristão, referente ao Curso de Especialização em Atenção à Saúde da Pessoa Idosa, Universidade Federal de Santa Catarina, 2016.

\section{RESUMO}

Objetivo: Identificar os riscos para o desenvolvimento de lesão por fricção (LF) em idosos longevos na atenção primária à saúde. Métodos: Pesquisa descritiva de abordagem quantitativa. Participaram 42 idosos com idade igual ou superior a 80 anos. Realizada entrevista estruturada e aplicado formulário. Realizada análise descritiva simples por meio da frequência absoluta e relativa das variáveis. Verificado o nível de significância entre os fatores clínicos de maior frequência e fatores sociodemográficos: idade, etnia e gênero, utilizando o teste qui-quadrado ou exato de Fisher. Efetuou-se o coeficiente de contingência com os parâmetros: $c \geq 0,750$ = associação forte; 0,500 a 0,749 = associação moderada; $\leq 0,499=$ associação fraca. Resultados: $\mathrm{A}$ idade variou de 80 a 100 anos, prevalecendo entre 80 e 85 anos (59,5\%), 28 (66,7\%) do sexo feminino, 38 (90.5\%) da raça branca e 30 (71,4\%) com primeiro grau incompleto. Em 41 idosos (97.6\%), observaram-se alterações dermatológicas relacionadas ao risco para LF (pele seca, mancha senil, equimose e edema). A idade foi o fator demográfico com maior associação aos achados clínicos ( $p=0,0397)$. Conclusão: Frente a esses achados, torna-se imperativa a necessidade de o enfermeiro munir-se de conhecimentos científicos, os quais permitem propor cuidados capazes de controlar os fatores de risco para desenvolvimento de LF.

DESCRITORES: Enfermagem geriátrica; Cuidados de enfermagem; Atenção primária à saúde; Idoso de 80 anos ou mais; Estomoterapia.

\footnotetext{
'Secretaria Municipal de Saúde de São José - Diretoria de Atenção Primária à Saúde - São José/SC - Brasil. ¿Universidade Federal de Santa Catarina - Departamento de Enfermagem - Florianópolis/SC - Brasil. ${ }^{3}$ Secretaria Municipal de Saúde de Florianópolis - Diretoria de Administração - Florianópolis/SC - Brasil. ${ }^{4}$ Universidade Federal de Santa Catarina - Programa de Pós-Graduação em Enfermagem - Florianópolis/SC - Brasil. Autor correspondente: Tatiana Martins | Avenida Madre Benvenuta, 40 - Santa Mônica | CEP: 88036-500 - Florianópolis/SC - Brasil | E-mail: tatiana_martins15@hotmail.com Recebido: Jun. 142018 | Aceito: Out. 202018
} 


\begin{abstract}
Objective: To identify the risks for the development of friction injury (FI) in long-lived elderly in primary health care. Methods: Descriptive research of quantitative approach. Participants were 42 elderly aged 80 years or more. Conducted structured interview and applied form. Simple descriptive analysis was realized by means of the absolute and relative frequency of the variables. Verified the level of significance between the most frequent clinical factors and sociodemographic factors: age, ethnicity and gender, using the chi-square test or Fisher's exact test. The contingency coefficient was performed with the parameters: $c \geq 0.750=$ strong association; 0.500 to 0.749 = moderate association; $\leq 0.499$ = weak association. Results: Age ranged from 80 to 100 years, prevailing between 80 and 85 years (59.5\%), 28 (66.7\%) women, 38 (90.5\%) white and 30 (71.4\%) with incomplete first degree. In 41 elderly patients (97.6\%), there were dermatological alterations related to the risk for FI (dry skin, senile blemish, ecchymosis and edema). Age was the demographic factor with the highest association with clinical findings $(p=0.0397)$. Conclusion: Faced with these findings, it becomes imperative that nurses be equipped with scientific knowledge, which allow them to propose care capable of controlling the risk factors for $\mathrm{Fl}$ development.
\end{abstract}

DESCRIPTORS: Geriatric nursing; Nursing care; Primary health care; Elderly with 80 years or more; Stomatherapy.

\title{
RESUMEN
}

Objetivo: Identificar riesgo del desarrollo de lesión por fricción (LF) en adultos mayores longevos en la atención primaria de salud Métodos: Estudio descriptivo de abordaje cuantitativo. Participaron 42 adultos mayores con edad igual o superior a los 80 años. Se realizó entrevista estructurada con aplicación de formulario. Realizado el análisis descriptivo simple por medio de la frecuencia absoluta y relativa de las variables. Verificado el nivel de significancia entre los factores clínicos de mayor frecuencia y factores sociodemográficos: Edad, etnia y género, utilizando el test exacto de Fisher. Se efectuó el coeficiente de contingencia con los parámetros: c $\geq 0,750$ = asociación fuerte; 0,500 a 0,749 = asociación moderada; $\leq$ 0,499 = asociación débil. Resultados: La edad varió de 80 a 100 años, prevaleciendo entre 80 y 85años (59,5\%), 28 (66,7\%) del sexo femenino, 38 (90.5 \%) de raza blanca y 30 (71,4\%) con primer grado incompleto. En 41 adultos mayores (97.6\%), se observaron alteraciones dermatológicas relacionadas al riesgo para LF (piel seca, mancha senil, equimosis y edema). La edad fue el factor demográfico con mayor asociación a los hallazgos clínicos ( $p=0,0397$ ). Conclusión: Frente a estos hallazgos, se vuelve imperativa la necesidad que el enfermero se capacite en conocimientos científicos, los cuales permitirán brindar cuidados capaces de controlar los factores de riesgo para el desarrollo de LF.

DESCRIPTORES: Enfermería geriátrica; Cuidados de enfermería; Atención primaria a la salud; Adulto mayor de 80 años o más; Estomoterapia..

\section{INTRODUÇÃO}

As lesões por fricção (LFs) são feridas decorrentes de trauma cutâneo ocasionado por fricção, cisalhamento e/ou contusão cuja tensão sobre a pele culmina em separação entre a epiderme e derme, ou de ambas as camadas, levando à formação de um retalho ${ }^{1,2}$.

Segundo o International Skin Tear Advisory Panel (ISTAP), as LFs podem ser classificadas de acordo com as características que apresentam, sendo o Skin Tear Classification System o instrumento mais recentemente desenvolvido com essa finalidade ${ }^{1}$. Esse instrumento foi adaptado culturalmente e teve sua viabilidade atestada no Brasil, sendo denominado Sistema de Classificação Star - Lesão por Fricção, permitindo classificar as LFs em categorias 1a, 1b, 2a, 2b e 3, com base na avaliação clínica da pele, cujo estadiamento varia de lesão com presença de retalho passível de realinhamento, sem alterações na coloração da pele, à perda completa do retalho ${ }^{3,4}$. Na maior parte dos casos, o surgimento da LF está associado ao sexo feminino, desidratação, condição nutricional prejudicada, mobilidade reduzida, alterações das funções sensorial e cognitiva, alterações dermatológicas, LFs anteriores, uso de fitas adesivas sobre a pele, problemas cardíacos, pulmonares e neuropáticos, polifarmácia, histórico de quedas, além de procedimentos traumáticos, como coleta de sangue. Contudo, observa-se forte relação com a idade, estando esse evento com frequência presente em idosos longevos (com mais de 80 anos de idade) $)^{1,2,5-7}$.

Não obstante, recentemente, um estudo de revisão sistemática identificou a prevalência de até 19,5\% dessas lesões no contexto domiciliar em diferentes países, sendo a longevidade um dos fatores de risco mais evidenciados ${ }^{7}$. Certamente a suscetibilidade da população longeva às LF deve-se à presença de alterações dermatológicas inerentes ao processo de envelhecimento, como a redução de secreções endócrinas, comprometimento da síntese de colágeno e da 
nutrição tecidual, além de alterações no tecido epitelial e conjuntivo $^{8}$ as quais interferem na manutenção da integridade da pele.

Nesse sentido, é notória a necessidade de se investir na produção de conhecimento, haja vista seu reflexo no aprimoramento do cuidado desempenhado pelo enfermeiro em sua prática assistencial, principalmente quando voltado à avaliação clínica da pele do idoso, a qual deve ocorrer de forma acurada, delineada por princípios cientificamente comprovados?.

Frente a essa problemática, considerando-se a magnitude desse fenômeno, justifica-se a relevância do presente estudo, pois a identificação dos fatores de risco para o desenvolvimento de LFs em idosos contribuirá para a elucidação de práticas de cuidado centradas na prevenção à ocorrência desse agravo, especialmente na população longeva, refletindo positivamente na qualidade de vida do idoso, bem como na redução do risco de complicações e da aplicação de recursos financeiros ao tratamento de lesões de pele potencialmente evitáveis. Consoante, para condução deste estudo, definiu-se, enquanto pergunta de pesquisa: quais fatores de risco estão associados à ocorrência de LFs em idosos longevos na comunidade?

\section{OBJETIVOS}

Identificar os riscos de desenvolvimento de LF em idosos longevos na atenção primaria à saúde.

\section{MÉTODOS}

Pesquisa descritiva, de abordagem quantitativa, desenvolvida com idosos residentes em um município do sul do Brasil.

Dessa forma, para condução da investigação, definiu-se, enquanto critérios de inclusão, idosos com idade igual ou superior a 80 anos, de ambos os sexos, cadastrados no território de abrangência da equipe de Estratégia Saúde da Família (e-SF) de uma Unidade Básica de Saúde (UBS), e/ou familiares/cuidadores do idoso para aqueles idosos os quais apresentassem condições clínicas que interferissem na condução do diálogo com o pesquisador ou quando manifestado por esse a necessidade de participação por parte de outrem. Logo, enquanto critérios para representação dos familiares e/ou cuidadores, considerou-se idade igual ou superior a 18 anos.

Conforme os dados do Sistema de Informação da Atenção Básica $(\mathrm{SIAB})^{10}$, base de dados utilizada pela Secretaria Municipal de Saúde no período da coleta de dados, a população de estudo correspondia a 76 idosos. Consoante, para o cálculo amostral, utilizou-se desvio-padrão de 10 unidades e nível de confiança de $95 \%$, sendo o tamanho recomendado para a amostra - 42 idosos - conforme apontado pelo sistema de Ensino-Aprendizagem de Estatística na Web (SEstatNet) proposto por Nassar et al. ${ }^{11}$.

Os dados foram obtidos no período de 5 de julho a 25 de agosto de 2016, por meio de entrevista estruturada, de forma aleatória, mediante visita domiciliar realizada com suporte dos agentes comunitários de saúde (ACS) do território. Durante a condução da entrevista, foram investigados aspectos sociodemográficos (idade, sexo, etnia, estado civil, grau de escolaridade, renda, além da presença ou não de cuidador), dados referentes à condição geral de saúde (aspectos nutricionais, uso de fármacos, presença ou não doenças crônicas não transmissíveis), hábitos de vida (ingesta hídrica), além da avaliação clínica da pele do idoso (avaliação quanto à presença de edema, equimose, pele seca e mancha senil).

Acrescenta-se que, diante da constatação de LF, essas foram avaliadas e classificadas de acordo com o Sistema de Classificação Star - Lesão por Fricção ${ }^{3}$.

Os dados foram transcritos por meio do software Formulários Google ${ }^{\circledR}$, sendo realizada análise descritiva simples por meio da frequência absoluta (n) e relativa (\%) das variáveis. Assim, verificaram-se o nível de significância entre os fatores clínicos evidenciados com maior frequência na amostra e os fatores sociodemográficos (gênero, idade e etnia) os quais configuram risco para ocorrência de LF.

A análise ocorreu por meio do software BioEstat 5.3, utilizando o teste qui-quadrado ou exato de Fisher quando menores que 5 o intervalo de confiança. Para verificar a magnitude de associação entre as variáveis, efetuou-se o coeficiente de contingência com os parâmetros: $c \geq 0,750=$ associação forte, 0,500 a $0,749=$ associação moderada e $\leq 0,499$ = associação fraca.

Vale ressaltar que, durante todo o estudo, foram respeitados os critérios com relação à pesquisa envolvendo seres humanos conforme a Resolução do Conselho Nacional de Saúde (CNS) 466/2012, tendo sido o estudo aprovado pelo Comitê de Ética em Pesquisa com Seres Humanos 
da Universidade Federal de Santa Catarina (UFSC) por meio do Parecer Consubstanciado 1.619.677, CAAE: 56298116.4.0000.0121.

\section{RESULTADOS}

No território de abrangência da e-SF, identificaram-se 76 idosos, porém 42 foram entrevistados por meio de visita domiciliar, considerando o recomendado pelo cálculo amostral. A faixa etária dos sujeitos variou de 80 a 100 anos de idade; a maior parte tinha idade entre 80 e 85 anos (59,5\%), 28 $(66,7 \%)$ do sexo feminino, 38 (90.5\%) brancos, 30 (71,4\%) com primeiro grau incompleto e 25 (59,5\%) viúvos. Quanto à renda, 38 (90.5\%) recebiam de um a três salários mínimos.

Do total, 24 (57.2\%) recebiam cuidados de profissional ou familiar e 18 (42.9\%) não tinham cuidador. Quanto aos aspectos nutricionais, $23(54,8 \%)$ apresentavam índice de massa corporal (IMC) dentro do considerado normal e 11 $(26,2 \%)$ com sobrepeso. Vale ressaltar que, para definição desses parâmetros, considerou-se: normal (valores entre 18,5 e $24,9 \mathrm{~kg} / \mathrm{m}^{2}$ ), baixo peso (inferior a $18,5 \mathrm{~kg} / \mathrm{m}^{2}$ ), sobrepeso (entre 25 e $29,9 \mathrm{~kg} / \mathrm{m}^{2}$ ) e obesidade (acima de $\left.30 \mathrm{~kg} / \mathrm{m}^{2}\right)^{12}$.

Quanto aos hábitos de vida, 25 (59,5\%) ingeriam quantidade igual ou inferior a 1 1/dia, $12(28,6 \%)$ de 11 a 1,5 1/dia e somente cinco acima de 1,5 L/dia.

Com relação ao histórico de saúde-doença, observou-se que $33(78,6 \%)$ idosos tinham hipertensão arterial sistêmica (HAS), nove $(21,4 \%)$ diabetes melito (DM) e seis $(14,3 \%)$ relataram ter tido acidente vascular encefálico (AVE) no último ano. Os demais tinham, em menor índice, outros tipos de doença, tais como cardiopatias, artrite, osteoporose, dentre outros.

Com relação ao uso de medicações contínuas, 39 (92,8\%) faziam uso de, no mínimo, uma medicação, sendo que, dessas, as mais frequentes eram: $18(46,2 \%)$ de losartana, $12(30,8 \%)$ de atenolol/carvedilol, $11(28,2 \%)$ de ácido acetilsalićlico e $10(25,6 \%)$ de metformina e estatinas. Apenas dois idosos relataram uso de antidepressivo e os demais faziam, em menor proporção, uso de outros grupos de medicamentos.

Durante avaliação clínica da pele dos participantes, constatou-se que 41 (97.6\%) tinham alterações dermatológicas das quais se configuram como fatores de risco para a ocorrência de LF: 35 (83,3\%) com pele ressecada, 10 (23.8\%) com mancha senil, sete (16.6\%) com edema e cinco (11.9\%) com equimose. Ademais, vale pontuar que, embora não apresentem relação com a ocorrência de LF, outras alterações dermatológicas também foram evidenciadas durante a investigação, quais sejam: dermatite, fissuras e micose (Tabela 1).

Tabela 1. Achados dermatológicos presentes na amostra ( $n=42)$, São José, estado de Santa Catarina, Brasil, 2016.

\begin{tabular}{ccc}
$\begin{array}{c}\text { Achados } \\
\text { dermatológicos }\end{array}$ & $\begin{array}{c}\text { Frequência } \\
\text { absoluta }(\mathrm{n})\end{array}$ & $\begin{array}{c}\text { Frequência } \\
\text { relativa (\%) }\end{array}$ \\
\hline $\begin{array}{c}\text { Pele ressecada } \\
\text { Mancha senil }\end{array}$ & 35 & 83,3 \\
\hline Edema & 70 & 23,8 \\
\hline Equimose & 5 & 16,6 \\
\hline Dermatite & 2 & 11,9 \\
\hline Micose & 2 & 4,7 \\
\hline Fissuras & 1 & 4,7 \\
\hline
\end{tabular}

Sobre os sinais associados avaliados, 18 (42.8\%) apresentaram alterações dermatológicas nos membros inferiores (MMII) e 19 (45.2\%) em MMII e membros superiores (MMSS). Da amostra investigada, apenas dois estavam em situação de restrição ao leito, sendo que 40 $(95,2 \%)$ deambulavam com auxílio.

Com relação à presença de LF, apenas um $(2,38 \%)$ apresentou lesão, sendo essa classificada como Categoria 3 na avaliação realizada por meio da versão adaptada para o Brasil do Star Skin Tears Classification System ${ }^{11,13,14}$. Verificou-se, também, outras três lesões, porém de etiologias distintas, as quais não se constituem em objeto de investigação do presente estudo.

Além dos resultados apresentados, os dados sociodemográficos foram associados aos fatores clínicos observados nos sujeitos do estudo.

\section{DISCUSSÃO}

Do total, 41 idosos apresentaram fatores de risco para ocorrência de LF, sendo sua frequência relativa expressa em $97.6 \%$ da população estudada. Consoante, a Tabela 1 evidenciou que os achados dermatológicos mais frequentes nos idosos eram pele ressecada, mancha senil, edema e equimose. Corroborando esses achados, pesquisadores apontaram que, em um grupo investigado ( $n=151)$, observou-se que o risco para LF era de duas a seis vezes maior em idosos que apresentavam equimose, mancha senil e edema ${ }^{13}$, tornando-se ainda maior nas 
extremidades (braços e pernas), uma vez que essas áreas estão mais expostas a agendes agressores ${ }^{2}$.

Vale ressaltar que a maior suscetibilidade do idoso a essas alterações possivelmente deve-se à redução dos tecidos responsáveis pelo acolchoamento subcutâneo, bem como sucessivas alterações vasculares ${ }^{14}$ provocados pelo processo de envelhecimento.

Além disso, em decorrência de alterações fisiológicas nas camadas da epiderme e derme, as quais interferem na capacidade de retenção de líquido nessa faixa etária, presume-se que esse seja um dos motivos pelo qual observou-se ressecamento cutâneo na população estudada ${ }^{8}$.

Um estudo realizado com idosos $(=250)$ ratificou que menos da metade dos participantes [121(48,4\%)] ingerira quantidade igual ou superior a dois litros de água diariamente ${ }^{15}$, sendo o parâmetro considerado pelos pesquisadores de, no mínimo, dois litros e, no máximo, dois litros e meio ao dia ${ }^{15}$. Acrescenta-se que a ingesta hídrica adequada seja fundamental para reduzir as chances de alterações decorrentes da desidratação ${ }^{15}$, tal qual o ressecamento cutâneo.

Há de se considerar, ainda, que a temperatura do inverno, estação do ano na qual se realizou a avaliação dos idosos deste estudo, pode ter contribuído para o surgimento desse achado. Estudiosos discorrem que, nesse período do ano, há redução da umidade do ar e da temperatura, o que ocasiona a redução da transpiração corporal, fazendo com que haja ressecamento cutâneo. No inverno, é muito comum que as pessoas tomem banhos mais quentes, $o$ que aumenta a redução da oleosidade natural da pele, diminuindo o manto lipídico que retém a umidade da pele e ocasionando ressecamento e resistência diminuída à agressão de substâncias alcalinas como o sabão ${ }^{8,16}$.
$\mathrm{Na}$ Tabela 2, observa-se que apenas a idade apresentou associação significativa ( $\mathrm{p}=0,0397)$ com os fatores clínicos, apontando, porém, baixa magnitude ( $\mathrm{c} \leq 0,499)$. Dessa forma, os achados do presente estudo, sobretudo no que concerne às alterações dermatológicas, seguramente fundamentam-se no descrito pela literatura ${ }^{1,2,5-8}$, porém, pode-se inferir que a baixa magnitude, no que concerne à idade, bem como a baixa associação das demais variáveis sociodemográficas aos fatores clínicos, possa estar relacionada à regionalidade da amostragem composta pelo território de adstrição de apenas uma e-SF.

No que diz respeito ao sexo, embora não observada associação significativa aos aspectos clínicos na amostra analisada, ressalta-se que o sexo feminino pode ser caracterizado como um fator de risco ${ }^{1}$ independente para ocorrência de LF. Possivelmente isso ocorre em decorrência de diferenças entre a pele do homem e da mulher, que podem deixá-la mais suscetível à ocorrência de agressões provocadas por cisalhamento, contusão e/ou fricção.

Vale pontuar que um estudo, cujo objetivo era analisar a influência da idade, sexo, cor e IMC na morfologia e imunidade local da pele, avaliou fragmentos de pele torácica de indivíduos autopsiados e concluiu que a epiderme de idosos do sexo masculino mostrou-se mais espessa, assim como com maior porcentagem de fibras elásticas, demonstrando maior proteção a agentes lesivos, destacando-se o trauma mecânico, em relação ao sexo feminino ${ }^{17}$.

A cor da pele também não apresentou relação significativa às variáveis clínicas, contudo, há de se considerar que a literatura sugere que a pele caucasiana apresenta maior risco para ocorrência de LF. Dentre outros aspectos, talvez esse fato se deva a maior vulnerabilidade da pele branca quando comparada à negra, a qual apresenta a camada da córnea mais

Tabela 2. Associação dos dados sociodemográficos aos fatores clínicos, São José, estado de Santa Catarina, Brasil, 2016.

\begin{tabular}{|c|c|c|c|c|c|c|c|c|c|c|c|}
\hline \multirow{3}{*}{ Dados } & \multicolumn{2}{|c|}{ Alterações nutricionais } & \multicolumn{6}{|c|}{ Fatores clínicos } & \multirow{3}{*}{$\chi^{2}$} & \multirow{3}{*}{ p-valor } & \multirow{3}{*}{ c } \\
\hline & Obeso & Baixo peso & $\begin{array}{c}\text { Doenças } \\
\text { cardiovasculares }\end{array}$ & Polifarmácia & Edema & $\begin{array}{l}\text { Pele } \\
\text { seca }\end{array}$ & $\begin{array}{l}\text { Mancha } \\
\text { senil }\end{array}$ & Equimose & & & \\
\hline & \multicolumn{8}{|c|}{$n(\%)$} & & & \\
\hline \multicolumn{12}{|l|}{ Sexo } \\
\hline Masculino & $7(50,0)$ & $1 *(7,1)$ & $10(71,4)$ & $3^{*}(21,4)$ & $3^{*}(21,4)$ & $12(85,7)$ & $6(42,8)$ & $2 *(14,2)$ & 6,745 & 0,455 & 0,215 \\
\hline Feminino & $8(28,6)$ & $2 *(7,1)$ & $25(89,2)$ & $21(75)$ & $5 *(17,8)$ & $21(75,0)$ & $9(32,1)$ & $4^{*}(14,2)$ & & & \\
\hline \multicolumn{12}{|l|}{ Idade } \\
\hline$\leq 85$ & $10(40,0)$ & $1 *(4,0)$ & $20(80,0)$ & $15(60,0)$ & $7(28,0)$ & $23(92,0)$ & $14(56,0)$ & $6(24,0)$ & 14,726 & 0,039 & 0,310 \\
\hline$>85$ & $5(29,4)$ & $2 *(11,7)$ & $15(88,2)$ & $9(52,9)$ & $0(0,0)$ & $10(58,8)$ & $1 *(5,8)$ & $0(0,0)$ & & & \\
\hline \multicolumn{12}{|l|}{ Etnia } \\
\hline Branco & $15(39,4)$ & $1 *(2,6)$ & $33(86,8)$ & $22(57,8)$ & $8(21,0)$ & $30(78,9)$ & $13(34,2)$ & $6(15,7)$ & & & \\
\hline Negro & $0(0,0)$ & $1 *(33,3)$ & $1 *(33,3)$ & $1 *(33,3)$ & $0(0,0)$ & $2^{*}(66,6)$ & $1 *(33,3)$ & $0(0,0)$ & 18,010 & 0,206 & $\begin{array}{r}0,338 \\
-\end{array}$ \\
\hline Pardo & $0(0,0)$ & $1 *(100,0)$ & $1 *(100,0)$ & $1 *(100,0)$ & $0(0,0)$ & $1 *(100,0)$ & $1 *(100,0)$ & $0(0,0)$ & & & \\
\hline
\end{tabular}


espessa. Logo, o aumento ou redução dessa camada interfere diretamente na integridade da pele, podendo comprometer seu mecanismo de proteção ${ }^{8,18,19}$.

Outra variável relevante refere-se ao perfil nutricional. Embora somente a idade tenha apresentado associação significativa com os critérios clínicos, torna-se importante elencar os aspectos nutricionais (e hídricos), haja vista serem esses citados como fatores de risco modificáveis ${ }^{20}$. Estudos apontam, como conclusão, associações independentes entre as alterações nutricionais e a prevalência do agravo, sendo essa elencada até mesmo como uma das principais questões relacionadas aos fatores de risco da $\mathrm{LF}^{21,22}$. Dessa forma, dada a crescente complexidade de tal critério, faz-se necessária sua avaliação no cenário de cuidados imediatos, a médio e longo prazos.

Convém ressaltar que também chamou atenção a presença de alterações cardiovasculares na população estudada, sobretudo HAS, expressa em uma parcela considerável de $33(78,6 \%)$ indivíduos. Atrelada a esse evento, atenta-se quanto à ocorrência de polifarmácia, capaz de repercutir em reações cutâneas devido às interações medicamentosas ${ }^{1}$. $\mathrm{Na}$ população investigada, ressalta-se o uso de anti-hipertensivos, os quais têm sido apontados, pela literatura, como classe predisponente à ocorrência de $\mathrm{LF}^{1}$.

Consoante, ressalta-se que, devido à instalação de condições crônicas (como, por exemplo, a HAS), podem ocorrer alterações no sistema circulatório capazes de comprometer a perfusão tissular e, consequentemente, a resistência da pele a lesões ${ }^{23}$. Igualmente, o uso de antihipertensivos também parece alterar a perfusão tecidual e comprometer a nutrição tissular, conforme apontado em um estudo de revisão ${ }^{24}$. Dessa forma, pode-se presumir que, na população investigada, essas condições potencializam o risco para ocorrência de LF, dada sua interferência na preservação da integridade da pele.

Ante aos achados do presente estudo, apontamos, como lacuna deste estudo, o número da população amostral e local de abrangência; entretanto, salienta-se que já estão em andamento novas pesquisas abrangendo maior número da população-alvo em diferentes realidades.

\section{CONCLUSÃO}

Os resultados do presente estudo evidenciaram que eram frequentes, na população estudada, fatores de risco para a ocorrência de LF, sendo a idade o fator demográfico com maior associação aos achados clínicos, possivelmente devido a grande parte das alterações presentes na pele do idoso estar relacionada ao curso do próprio processo de envelhecimento.

Dentre as alterações dermatológicas de maior relevância, evidenciou-se a pele ressecada, a mancha senil, o edema e a equimose como mais frequentes, sendo essas condições potencialmente evitáveis por meio de cuidados de enfermagem.

O sexo e a cor da pele não apresentaram associação estatística significativa na população investigada, contudo, a literatura sugere que essas variáveis, de forma independente, exercem influência no desenvolvimento de LF.

Ante aos esses achados, emoldura-se a necessidade de o enfermeiro munir-se de conhecimentos científicos os quais o permitam propor cuidados capazes de refletir no extinguir, minimizar, controlar os fatores de risco para o desenvolvimento de LF, sendo fundamental o monitoramento de condições associadas àqueles não modificáveis, como, por exemplo: idade, sexo e etnia.

Diante do exposto, é indispensável a atuação do profissional enfermeiro no âmbito da atenção primária à saúde enquanto educador em saúde, uma vez que suas práticas de cuidado são capazes de prevenir condições de morbidade, promovendo maior qualidade de vida ao idoso.

\section{CONTRIBUIÇÃO DOS AUTORES}

Conceitualização, Tristão FR e Girondi JBR; Metodologia, Tristão FR; Girondi JBR e Lima DKS; Investigação, Tristão FR; Girondi JBR e Lima DKS; Redação - Primeira versão, Tristão FR; Girondi JBR; Hammerschmidt KAS; Soares CF; Martins T e Lima DKS; Redação - Revisão \& Edição, Tristão FR; Girondi JBR; Hammerschmidt KAS; Soares, CF e Martins T; Supervisão, Girondi JBR. 


\section{REFERÊNCIAS}

1. Leblanc K, Baranoski S, Christensen D, Langemo D, Sammon MA, Edwards K, et al. International Skin Tear Advisory Panel: a tool kit to aid in the prevention, assessment, and treatment of skin tears using a simplified classification system. Clin Manag. 2013; 26(10):459-76. https://doi.org/10.1097/01. ASW.0000434056.04071.68.

2. Clothier A. Assessing and managing skin tears in older people. Independent Nurse. 2014;12(8):23-6. https://doi. org/10.12968/indn.2014.8.23.

3. Strazzieri-Pulido KC, Santos VLCG, Carville K. Adaptação cultural, validade de conteúdo e confiabilidade interobservadores do "STAR Skin Tear Classification System". Rev Latino-Am Enfermagem. 2015;23(1):155-61. https://doi. org/10.1590/0104-1169.3523.2537.

4. Strazieri-Pulido KC. Adaptação cultural e validação do instrumento "Star Skin Tear Classification System" para a língua portuguesa no Brasil [dissertação]. São Paulo (SP): Universidade de São Paulo, Escola de Enfermagem; 2010.

5. O'Regan A. Skin tears: a review of the literature. World Counc Enteros Ther J. 2002;22(2):26-31.

6. Oliveira GG, Neves BB, Jorge LB, Neris JCD, Rauber BR, Caberlon IC, et al. Diferenças na gravidade da queda entre idosos jovens e longevos. PAJAR. 2016;4(2):54-9. https://doi. org/10.15448/2357-9641.2016.2.26181.

7. Strazzieri-Pulido KC, Peres GRP, Campanili TCGF, Santos VLCG. Prevalência de lesão por fricção e fatores associados: revisão sistemática. Rev Esc Enferm USP. 2015; 49(4): 67480. https://doi.org/10.1590/S0080-623420150000400019.

8. Fortes $T M L$, Suffredini IB. Avaliação da pele em idosos: revisão da literatura. J Health Sci Inst. 2014; 32(1):94-101.

9. Amante LN, Girondi JBR, Carmargo RCRM, Nascimento KC, Knihsel NS. Cuidado de enfermagem no período perioperatório: intervenções para a prática. Vol 1. Curitiba: CRV; 2015.

10. Prefeitura de São José. Relatório de Produção SIAB 2015. São José: Secretaria de Saúde; 2015.

11. Nassar SM, Wronscki VR, Ohira M, et al. SEstatNet - EnsinoAprendizagem de estatística na Web [Internet]. Florianópolis: SEstatNet; 2018 [acesso 2018 Sep 09]. Disponível em: http:// sestatnet.ufsc.br.

12. Centro Brasileiro de Informação sobre Medicamentos. BVS. Obesidade [Internet]. Brasília: Ministério da Saúde; 2018 [acesso 2018 Out 18]. Disponível em: http://bvsms.saude. gov.br/bvs/dicas/215 obesidade.html/.

13. Lewin GF, Newall N1, Alan J, Carville KJ, Santamaria NM, Roberts PA. Identification of risk factors associated with the development of skin tears in hospitalised older persons: a case-control study. Int Wound J. 2016;13(6):1246-51. https:// doi.org/10.1111/iwj.12490.
14. Hammerschmidt KSA, Tier CG, Anhaia L, Santos SSC, Paz $L$, Ramos AK et al. Cuidados de enfermagem na saúde do idoso. In: Cuidado de enfermagem: interfaces teóricas e práticas no ciclo vital do ser humano. Curitiba: CRV; 2015. p. 261-308.

15. Garbaccio JL, Ferreira AD, Pereira ALGG. Conhecimento e prática referidos por idosos no autocuidado com a pele no centro-oeste de Minas Gerais. Rev Bras Geriatr Gerontol 2016;19(1):45-56. https://doi.org/10.1590/18099823.2016.14237.

16. Sociedade Brasileira de Dermatologia. Cuidados com a pele no inverno [Internet]. Rio de Janeiro: SBD; 2016 [acesso 2016 Set 09]. Disponível em: http://www.sbd.org.br/ cuidados/cuidados-com-a-pele-no-inverno/.

17. Oliveira LF. Avaliação morfológica e imunológica da pele de acordo com as características epidemiológicas de idosos autopsiados [dissertação]. Uberaba (MG): Universidade Federal do Triângulo Mineiro; 2011.

18. Le Blanc K, Baranoski S. Skin tears: state of the science: consensus statements for the prevention, prediction, assessment, and treatment of skin tears. Adv Skin Wound Care. 2011;24(9):2-15. https://doi.org/10.1097/01. ASW.0000405316.99011.95.

19. Bianch J. Preventing, assessing and managing skin tears. Nurs Times. 2012;108(13): 12-6.

20. LeBlanc K, Baranoski S. Skin Tears: finally recognized. Adv Skin Wound Care. 2017;30(2):62-3. https://doi.org/10.1097/01. ASW.0000511435.99585.0d.

21. Munro EL, Hickling DF, Williams DM, Bel J. Malnutrition is independently associated with skin tears in hospital inpatient setting - Findings of a 6-year point prevalence audit. Int Wound J. 2018;15(4):527-33. https://doi.org/10.1111/ iwj.12893.

22. Serra R, lelapi N, Barbetta A, Franciscis S. Skin tears and risk factors assessment: a systematic review on evidencebased medicine. Int Wound J. 2018;15(1):38-42. https://doi. org/10.1111/iwj.12815.

23. Souza NR, Freire DA, Souza MAO, Melo JTSM Santos LV, Bushatsky M. Fatores predisponentes para o desenvolvimento da lesão por pressão em pacientes idosos: uma revisão integrativa ESTIMA, Braz. J. Enterostomal Ther 2017;15(4):229-39. https://doi.org/10.5327/Z18063144201700040007.

24. Almeida AFS, Soares TSB, Abreu RNDC, Mendonça FAC, Guanabara MAO, Sampaio LRL. Influência de fármacos sobre a formação sobre a formação de úlceras por pressão: revisão integrativa. Revista Enfermagem Contemporânea. 2016;5(1):118-24. https://doi.org/10.17267/2317-3378rec. v5i1.681. 